\title{
The several Cs of translational clinical research
}

\author{
David G. Nathan
}

Harvard Medical School, Dana-Farber Cancer Institute, Boston, Massachusetts, USA.

\begin{abstract}
Perhaps because I am a veteran of the "good old days" (they were really quite bad), young physicians who hope to become clinical investigators often ask me how they might establish their careers. Many are more than a little worried about their futures and often have trouble envisioning a career path that is financially secure for themselves and their families. The grumbling of clinical investigators a few years their senior enhances their angst. So I try to encourage these young physicians because I know the great intellectual (if not monetary) rewards of the field and because I know that the future of medicine absolutely depends on clinical investigators. The following is what I try to say to them.
\end{abstract}

First I try to point out that the definition of clinical research is very broad. It encompasses clinical trials, outcomes, health delivery, epidemiological, and psychosocial research (1). Though many experts seem to believe that the term "clinical research" is restricted to those areas, nothing could be further from the truth. One of the most important, and most difficult, areas of clinical research is currently called (for want of a better term) translational clinical research. Translational clinical research focuses on the bench-to-bedside interface and requires a physician-investigator schooled in the clinical aspects of a subspecialty, skilled in biomedical science and its methodologies, and endowed with enough intuition to recognize the patients who can, if carefully investigated in the laboratory, reveal the nature and/or appropriate treatment of their diseases. During this process the investigator may uncover fundamental rules of biology (2). It is this class of clinical investigator that has seemed most endangered since James Wyngaarden, a former director of the $\mathrm{NIH}$, first called attention to its fragility (3).

Translational clinical investigators come in at least two flavors, best described by Joseph Goldstein and Michael Brown (4). Both classes likely consist of physicians. One class includes physicianscientists interested in a disease mechanism and even occasionally interested in seeing patients. But these almost never interact in their research with an intact patient/subject. Such diseaseoriented researchers are content to study tissue samples, cell lines, and model systems such as mice, fish, and yeast and do so with great benefit. They are to be encouraged, praised, loved, and supported, but they are not real clinical researchers. Their career paths are only slightly distinguishable from those of basic scientists (who should also be well supported).

The other class of physician-scientists includes patient-oriented researchers. They actively search for patients who may enable them to uncover the secrets of complex diseases, care for those patients, and, with their permission, undertake to explore new diagnostic and therapeutic approaches to treating their diseases. Such patient-oriented clinical investigators are obviously challenged by the growing complexities of clinical medicine and biomedical science because at least a decade of intensive work is required to come close to mastery of the fundamentals.

Nonstandard abbreviations used: AHC, academic health center; POTCI, patientoriented translational clinical investigator

Conflict of interest: The author has declared that no conflict of interest exists.

Citation for this article: J. Clin. Invest. 115:795-797 (2005).

doi:10.1172/JCI200524753.
Much of the angst that I hear from novitiate patient-oriented translational clinical investigators (POTCIs) relates to the enormous amount of time in penurious circumstances that is required to achieve sufficient training and experience before they can hope for any real independence. They are usually in their mid-twenties when they graduate from medical school, approaching thirty when they complete a residency in a specialty, and in their mid-thirties when they have acquired the necessary scientific skills to conduct an independent laboratory-based inquiry. During this lengthy and impecunious training experience, they are often struggling to pay off a crushing medical school debt, and their spouses and parents are regularly asking them when they will be gainfully employed. Their worries are exacerbated by fears of failure. Will they actually have the insight that they must exhibit if they are to find and study the patient or patients who will enable them to uncover the secrets of a human disease? Will they have the skill to learn something useful about those patients at the bench? These understandable concerns, coupled with realistic worries about the future of their families, may drive them toward more familiar and more immediately rewarding ground - the full-time care of patients.

The policies of the NIH extramural programs have been regularly assailed when concerns are voiced about the paucity and fragility of POTCIs. But the ranting is quite misplaced. We, the members of NIH review groups, make the decisions regarding investigator and trainee support by the NIH. We collectively decide what we will recommend for funding, and if our voices are not strong enough in review meetings, we have only ourselves to blame. Priority score voting is surely not a perfect procedure, and herd instincts can dominate the decisions. But a determined spokesperson for an excellent POTCI candidate (assuming POTCIs are not too discouraged to apply) can make a big difference. Furthermore, the NIH has been responsive to our calls for more support for clinical researchers, including POTCIs, and has tried to improve the balance of study sections. The latter depends, of course, on the willingness of experienced clinical researchers to serve on such panels. A series of new NIH-initiated training and support grants in the $\mathrm{K}$ series has provided the means for teaching clinical research methods for many medical schools, support for young investigators who have completed their fellowship training, support for mentors of such investigators, and major debt relief for physicians who elect a patient-oriented clinical research career (5). It is not clear that the NIH can do much more right now, particularly while its budget heads into temporary doldrums.

What can academic health centers (AHCs) do to alleviate the stress in the lives of the POTCIs on whom they must rely to carry vital information back and forth between bench and bedside? First 
they can examine their own financial commitments: AHCs scarcely operate in poverty. Many are money-making organizations if they are well run and have a viable patient base because they have mastered the art of dealing with managed care. That is one reason, albeit relatively small, that the cost of medical care is accelerating at an alarming rate. AHCs are doing so well that they are physically expanding - the national bird of academic medicine seems to be the crane. If AHCs can afford new beds, new labs, and new mouse and even zebrafish facilities, they can certainly afford core support for clinical researchers, including POTCIs. They can pay for the statisticians, nurse clinicians, and data managers who are vital members of a clinical research team. They can establish core labs in order to provide crucial experimental data for a group of investigators. And they can help physician-investigators through the toils of the protocol and institutional review board mazes that make clinical research seem like an endless run through a gauntlet of cunctators (spiritual descendants of Roman Emperor Quintus Fabius Maximus the Delayer). Most of all, AHCs can engender a culture of collaboration in clinical research, particularly the research carried out by POTCIs.

It is important to expand on collaboration because I believe that it may be the most important ingredient of all. Today's young POTCIs know that they cannot emulate the POTCIs of yesteryear because they cannot possibly establish the same mastery of the science and clinical medicine of the twenty-first century that was exhibited by those now-hoary greats of the 1950s and 1960s. The reason is obvious: the necessary knowledge bases have expanded beyond the capacity of normal mortals. Individuals must make choices. If laboratory-based clinical research is to be their métier, they may have to tolerate some atrophy of their general clinical skills in favor of mastery of the clinical knowledge that is relevant to the research specialty that stimulates them. Attention to the accrual of clinical knowledge and experience within a specialized area will eventually render the POTCI less capable of brilliant clinical decisions in infrequently trampled fields. A budding POTCI needs to focus on the care of patients who are part of his or her research program; however, as his or her formerly broad medical knowledge straitens, the once all-comprehending POTCI will likely be forced to cede control of some medical decisions to full-time clinical collaborators when the patient develops a problem beyond the POTCI's expertise. In sum, POTCIs are likely to retain enough clinical knowledge and instinct and accrue enough experience to work their way through to a broad-brush solution of a complex clinical problem in another field, but they will often wish to collaborate with a full-time clinician to establish the details of care and offer the optimal outcome to a trusting patient.

In order to maintain their cutting-edge investigative skills, POTCIs may also wish to collaborate with basic scientists who are completely devoted to laboratory efforts. The rapid pace of biomedical science will demand not only these collaborations, but frequent sabbaticals in basic science laboratories. AHCs will need to have the wisdom to part temporarily with their precious POTCIs for such sabbaticals. Investment in the continued scientific education of this important class of clinical researchers is apt to reap important dividends.

Though collaboration is an obvious necessity, the published results of collaborative efforts may be problematic. If the position of one's name on a paper is to remain the currency of success, a natural collaborative effort may be fraught with obstructive tension because, in many AHCs, promotion committees tend to ignore any paper in which the candidate is neither first nor last author. Such a rigid view of biomedical accomplishment scarcely enhances a collaborative culture. AHCs need to rid themselves of the author-position albatross and substitute an honest evaluation of an author's real contribution to a published piece of science. Then they can develop a portfolio of a candidate's contributions and make a sensible decision about promotion. We are currently initiating that process at Harvard Medical School's affiliated hospitals and we hope eventually to banish the albatross.

AHCs can also help the POTCI cause by focusing major effort on the training of medical students in clinical research. I have trained a fairly large number of physician-scientists, and to my satisfaction, many of them are successful POTCIs. But I have rarely seen a POTCI emerge who had not been bitten by the laboratory bug in medical school. In fact, I would never have become a POTCI if I had not had a very satisfying research experience in medical school. Today many medical students take time off to explore research experiences. At least a quarter of Harvard's medical students delay their graduation in order to pursue research. But too few have research training experiences that involve patients, just as $\mathrm{MD} / \mathrm{PhD}$ training experiences almost never involve patients. AHCs need to change that culture. It is easy to dump a student into a basic research laboratory and hope for the best. It is much harder to establish an experience in which a student is mentored by an excellent scientist in a laboratory that is specifically committed to basic understanding of a human disease and mentored just as carefully by a full-time clinician in a clinic that is devoted to the treatment of that disease. In the clinic, the student will find and help to care for the patients who have the cells that the student will study in the laboratory. That sort of experience is difficult to arrange. The full-time scientist and the clinician need to work out the project well in advance of the student's arrival on the scene. Both the scientist and the clinician must take the time to ensure success. Such careful and time-consuming planning does not guarantee the success of the project or the student's subsequent enthusiasm for a research career, but it markedly enhances the chances of a successful outcome. We will enlarge the pool of future POTCIs if we focus on medical students and point them toward that niche. To do so, AHCs will have to find a way to support the mentors who make it possible. The Doris Duke Charitable Trust has developed an important granting program that supports AHCs to pursue clinical research training for medical students.

In my discussions with young aspirants to a POTCI career, I often review what I now call "the several Cs of clinical research." The most important $\mathrm{C}$ is clinical focus. A patient-oriented investigator must be primarily interested in a particular disease and the patients who suffer from it. This interest may lead (and, in fact, often leads) to a basic biological inquiry, but the driving force is the patient. Collaboration has already been discussed. A successful POTCI must be a collaborator with basic scientists and full-time clinicians. That triumvirate is essential for success. Courage is a sine qua non, and by courage I do not mean confidence, although no one can achieve anything without confidence. I mean the courage to learn new techniques and new approaches to the hidden mysteries of illness. POTCIs fail when they slavishly adhere to a set of painfully learned techniques and look for ways to apply them. Goldstein described that fatal condition as "paralyzed academic investigator's disease syndrome" (6). Critical awareness of the literature and the field is an absolute requirement. That means travel to meetings where the "hot" new information is emerging. Travel is costly and it attenuates family life, but it is necessary. A constructive infrastructure is essential. The AHC must 
provide the support staff and core labs that permit any kind of clini$\mathrm{cal}$ research to be pursued. Cooperative spouses are critical. Medical schools are breeding grounds for dual careers. The couple must find a way to share responsibility for child rearing. Women are interested in POTCI careers, but they cannot achieve success unless they and their similarly stressed spouses can establish a functional and safe environment for their children. A simple example is the fact that important research conferences occur at times when day care centers are closed. It's important for POTCIs to attend those conferences. AHCs need to grapple with that problem and develop excellent day care centers that remain open long enough to permit POTCI mothers or fathers to work effectively, secure in the knowledge that their children are well cared for. How best to do that is itself a valuable subject for research.

The patient's consent to participate in clinical research has become a cottage industry for lawyers. The forms that patients sign often bear no resemblance to ordinary communication between humans. A POTCI must learn to converse in normal terms with a patient and write a note in the chart that reflects that conversation. The signed form is only step one in the process. The value of a detailed note that describes the consent process becomes obvious if an adverse outcome leads to an unwanted legal proceeding.

Conflict of interest is a deadly bacillus. A physician who accepts a personal check from or owns stock in a company that produces a drug or a device that the physician is investigating in his or her patient is involved in an unacceptable conflict of interest. If anything goes wrong, the press and Congress will ensure that the physician and his or her institution will never hear the end of it. AHCs must thoroughly eschew such conflicts.

Those who hope to achieve success in biomedical science must avoid the menace of the "chronophage." These are well-meaning supervisors who ask eager-to-please young people to perform extraneous tasks that eat their time. Patient-oriented translational clinical research is difficult and requires almost complete attention. Aspiring POTCIs can be particularly susceptible to the wiles of the chronophage because they do not like to say no. Women and minorities are particularly vulnerable because, until recently, there have been relatively few of them, and they are constantly sought for membership on committees in order to maintain an appearance of diversity. I advise POTCIs to develop a hearing disorder and stay away from committees or heavy teaching duties while they are developing their careers. I know of what I speak because I am an expert chronophage myself.

Caring mentors are the real key to success. A young POTCI must have a great mentor in the lab and in the clinic. The mentor should try to be very available, steer the POTCI into the most productive areas and away from barren or overworked soil, solve inevitable conflicts and jealousies, help to find collaborating experts, and seek little or no credit but nevertheless find joy from the POTCI's success. I know that joy. It is entirely worth the effort.

There are certainly other important aspects of POTCI care and nurturing that could be productively explored. But the essential point is that the development of this class of medical researchers is a serious responsibility of academic medicine. If we are to translate the fruits of biomedical research and ensure that they lead to the improved care of patients, we must produce and nurture these physicians. If we fail to do so, Congress will lose interest and all of the NIH portfolio will be weakened because Congress responds to the plight of real people. Its members understand that the study of zebrafish is important, but only if it leads to improved care of their constituents. We have to focus on our POTCIs and ensure their success.

\section{Acknowledgments}

The author is grateful to Samuel E. Lux IV and Edward J. Benz Jr. for helpful criticism and to Toby Church for skilled assistance.

Address correspondence to: David G. Nathan, Harvard Medical School, Dana-Farber Cancer Institute, 44 Binney Street, Boston, Massachusetts 02115, USA. Phone: (617) 632-2155; Fax: (617) 632-2161; E-mail: david_nathan@dfci.harvard.edu.

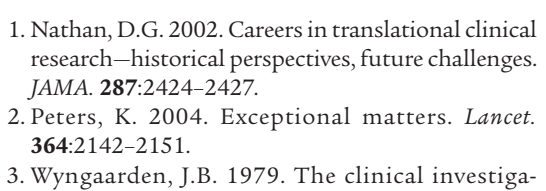
tor as an endangered species. N. Engl. J. Med. 301:1254-1259.

4. Goldstein, J.L., and Brown, M.S. 1997. The clinical investigator: bewitched, bothered, and bewildered - but still beloved. J. Clin. Invest. 99:2803-2812.

\footnotetext{
5. Nathan, D.G., and Wilson, J.D. 2003. Clinical research and the NIH - a report card. N. Engl. J. Med. 349:1860-1865.

6. Goldstein, J.L. 1986. On the origin and prevention of PAIDS (Paralyzed Academic Investigator's Disease Syndrome). J. Clin. Invest. 78:848-854.
} 